\title{
A Favorable Response to Levetiracetam in a Patient with Metastatic Adenoid Cystic Carcinoma
}

\author{
Shinya Sakata ${ }^{1}$, Sho Saeki ${ }^{1}$, Yasuhiro Terasaki ${ }^{2}$, Yoshihiro Natori ${ }^{3}$ and Kazuhiko Fujii ${ }^{1}$
}

\begin{abstract}
:
Adenoid cystic carcinoma (ACC) is a rare cancer, and there are no standard-of-care treatments for patients with metastatic ACC. We herein report a patient with lung metastasis of ACC who achieved a favorable response to levetiracetam. A 52-year-old Japanese man was admitted to our hospital because of multiple lung metastases of ACC. We performed first-line chemotherapy with cisplatin plus gemcitabine, and subsequently oral S-1 as second-line chemotherapy, which resulted in disease progression. The patient developed symptomatic epilepsy and received levetiracetam ( $250 \mathrm{mg}$ twice daily). At five months after the initiation of levetiracetam, chest computed tomography showed regression of the metastatic lung lesions.
\end{abstract}

Key words: adenoid cystic carcinoma, levetiracetam, lung metastasis, regression

(Intern Med 57: 721-724, 2018)

(DOI: 10.2169/internalmedicine.9300-17)

\section{Introduction}

Adenoid cystic carcinoma (ACC) is a rare cancer that occurs mainly in the salivary glands. ACC accounts for approximately $1 \%$ of all head and neck malignancies (1). Because of its rarity and clinical features, there are fewer evidence-based therapies for recurrent or metastatic ACC compared than other cancers. A novel, effective, evidencebased treatment for recurrent or metastatic ACC is needed.

Levetiracetam, an antiepileptic drug that belongs to the group of non-enzyme-induced antiepileptic drugs, has been reported to have fewer side effects than traditional antiepileptic drugs and to have no effect on the kinetics of other drugs. To our knowledge, the antitumor activity of levetiracetam against ACC has not been reported.

We herein report the case of a patient with lung metastasis of ACC who achieved a favorable response to levetiracetam.

\section{Case Report}

A 52-year-old Japanese man with a 4-year history of ACC was admitted to our hospital because of multiple lung me- tastases. Four years earlier, he had undergone radical resection of ACC in the left lacrimal gland followed by postoperative radiotherapy. The patient denied having a smoking history. There was no family history of cancer. A clinical examination initially showed no abnormal findings. The results of laboratory examinations were normal. Chest computed tomography (CT) revealed multiple lung nodules (Fig. 1A).

We diagnosed recurrence of ACC and initiated anti-cancer chemotherapy with a 3-week regimen of cisplatin $(80 \mathrm{mg} /$ $\mathrm{m}^{2}$, day 1) plus gemcitabine $\left(1,000 \mathrm{mg} / \mathrm{m}^{2}\right.$, days1 and 8$)$ for 4 cycles. However, after 4 cycles of chemotherapy, CT demonstrated progression of the metastatic lung lesions. As second-line chemotherapy, the patient received oral S-1 (40 $\mathrm{mg} / \mathrm{m}^{2}$ twice daily for 2 weeks) repeated every 3 weeks for 4 cycles, and this resulted in disease progression of the metastatic lung lesions (Fig. 1B). Subsequently, the patient received the best supportive care, as no effective chemotherapy regimen remained.

Two years later, chest CT showed progression of the lung metastases (Fig. 1C). The lung metastases gradually progressed during the observation period. Subsequently, head contrast magnetic resonance imaging (MRI) revealed two metastases in the patient's brain (Fig. 2). Stereotactic radio-

${ }^{1}$ Department of Respiratory Medicine, Kumamoto University Hospital, Japan, ${ }^{2}$ Department of Analytic Human Pathology, Nippon Medical School, Japan and ${ }^{3}$ Department of Neurosurgery, Iizuka Hospital, Japan

Received: April 3, 2017; Accepted: July 5, 2017; Advance Publication by J-STAGE: November 20, 2017

Correspondence to Dr. Sho Saeki, saeshow@wg7.so-net.ne.jp 

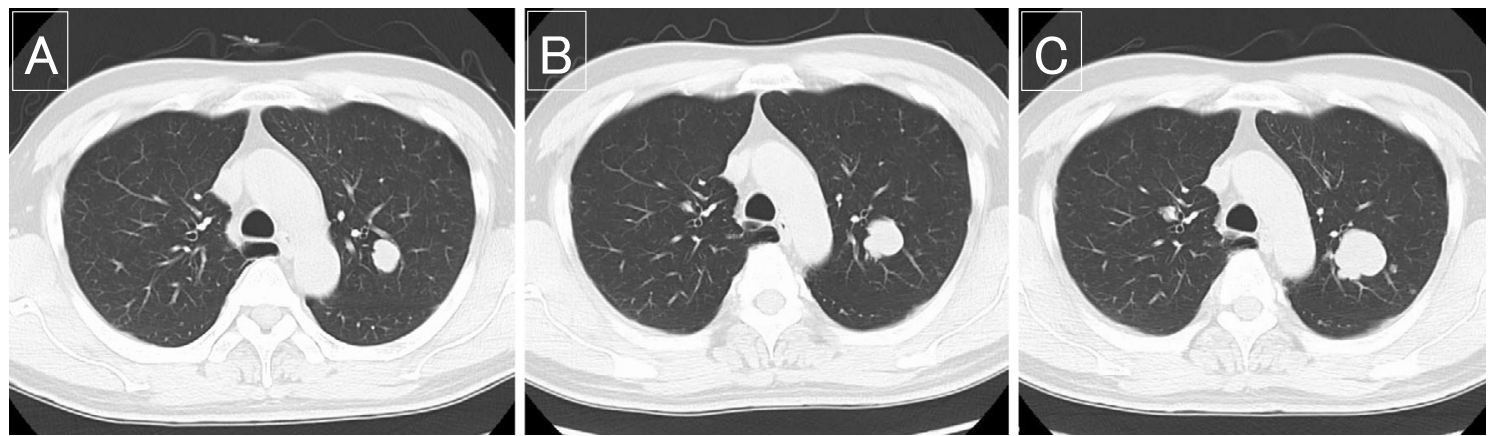

Figure 1. A: Chest CT demonstrating a nodule in the left patient's upper lung before chemotherapy. B: After four cycles of second-line chemotherapy, the left upper lung nodule showed progression. C: At two years after the last chemotherapy administration, the upper lung mass showed progression.
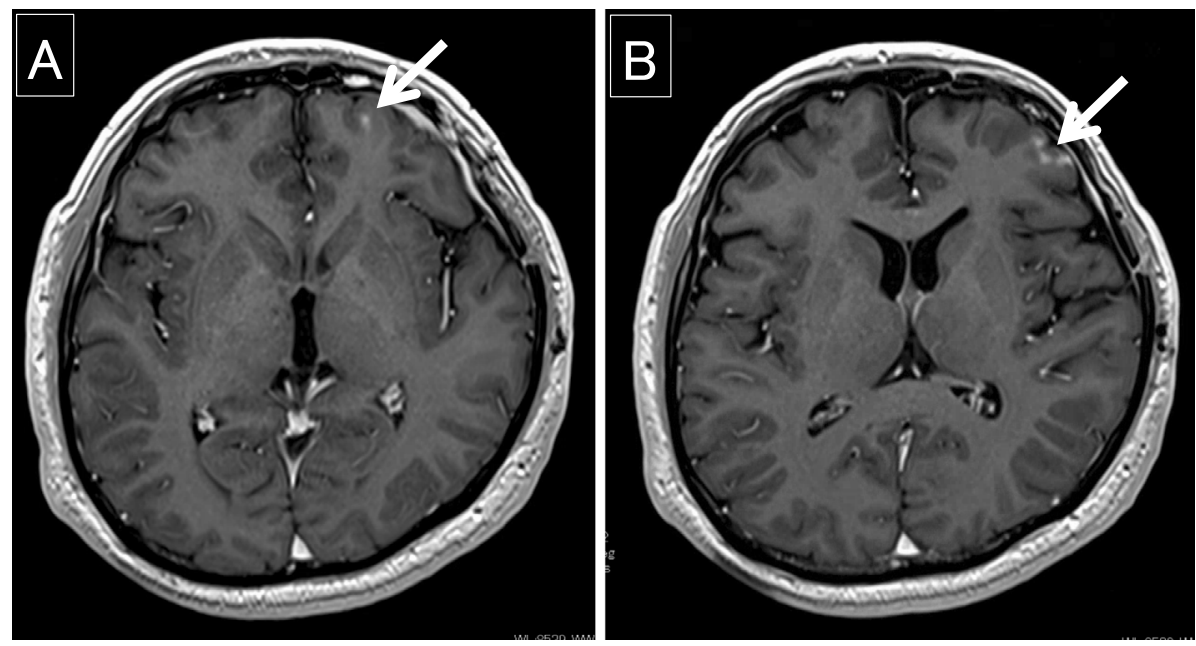

Figure 2. A, B: Head contrast MRI showing two brain metastases (arrow).

therapy was administered, and the brain metastases showed regression. However, symptomatic epilepsy developed three months after the completion of the stereotactic radiotherapy.

Four years after the last chemotherapy administration, the frequency of the patient's epilepsy seizures had increased; he was treated with valproic acid (400 mg twice daily). However, the persistence of simple partial seizures was observed, and we administered levetiracetam (250 mg twice daily). At five months after the initiation of levetiracetam, chest CT showed regression of the metastatic lung lesions (Fig. 3A, B, D and E). However, at the evaluation performed 27 months after the initiation of levetiracetam, chest CT demonstrated tumor progression (Fig. 3C and F).

\section{Discussion}

ACC is regarded as an aggressive, often indolent tumor with a recurrence rate of $40-50 \%$ after curative intent treatment (such as radical resection followed by postoperative radiotherapy) (2). Regional and distant recurrence of ACC is relatively common, mainly to the lung, bone and liver (3). ACC is refractory to chemotherapy, and the impact of chemotherapy on the survival of ACC patients is unclear. There- fore, there are no standard-of-care treatments for patients with metastatic ACC.

Cytotoxic agents commonly administered as monotherapy for the treatment of ACC are cisplatin, vinorelbine, paclitaxel, epirubicin and gemcitabine. However, few of these cytotoxic agents have shown efficacy. In their review, Laurie et al. noted that the mean survival of patients with metastatic ACC was 11 months, and a few cases showed an objective response (i.e. a partial or complete response) with chemotherapy (4). A study that examined gemcitabine alone showed no objective responses, progressive disease (PD) in $38 \%$ of the patients, and stable disease (SD) in $52 \%$ of the patients (5). However, in a report by Laurie et al., a phase II study of platinum and gemcitabine combination therapy showed objective responses in $20 \%$ of advanced ACC cases (6). Hiraga et al. reported a case of successful treatment of metastatic ACC with S-1 (7).

Although the standard chemotherapy for ACC has not been established, for our present patient, we selected cisplatin plus gemcitabine as first-line chemotherapy and S-1 as second-line therapy with reference to the above reports. Because of the low rate of response to cytotoxic chemotherapy for ACC, molecular-targeted therapies have been admin- 


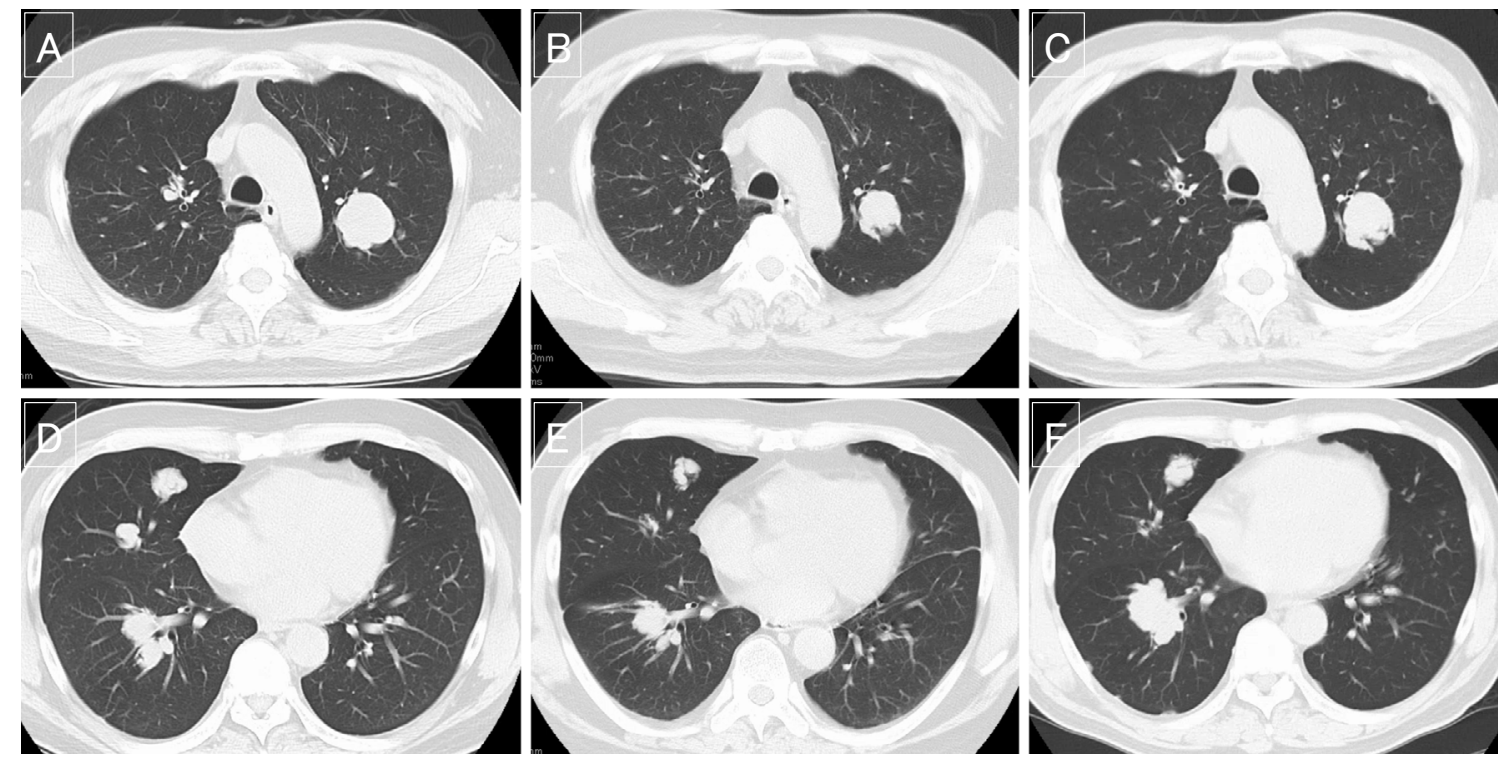

Figure 3. A, D: Chest CT demonstrating a mass in the patient's left upper lung and right middle and lower lung before the initiation of levetiracetam. B, E: At five months after the initiation of levetiracetam, the left upper lung mass and the right middle and lower lung nodules showed regression. C, F: Chest CT at 27 months after the initiation of levetiracetam demonstrated progression of the mass in the left upper lung and the right middle and lower lung.
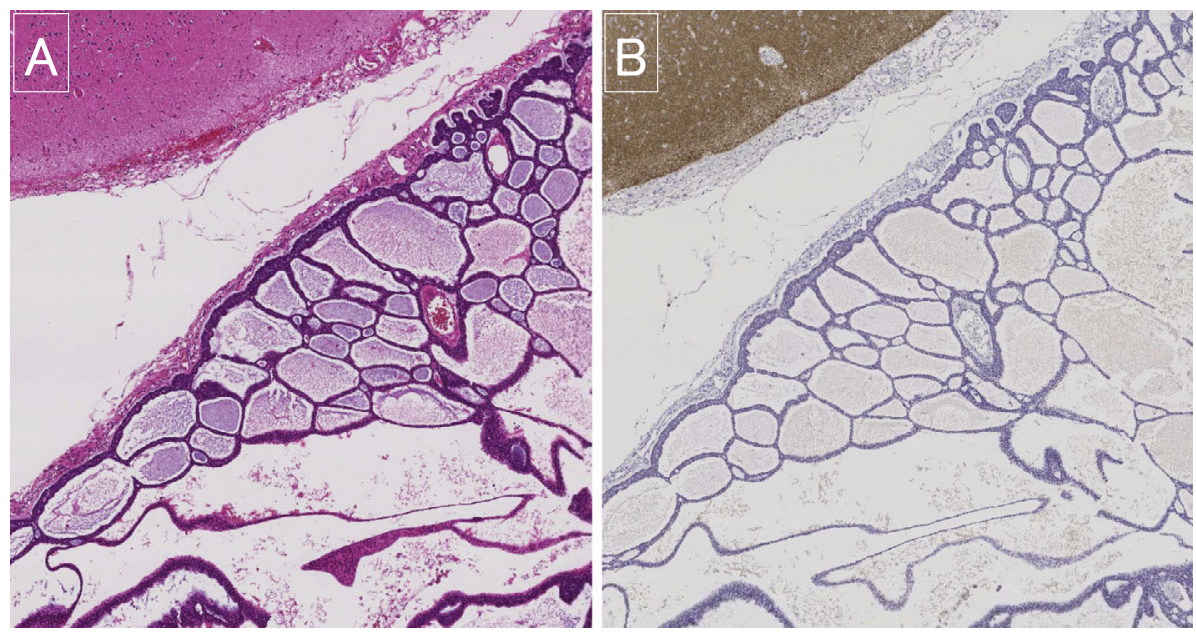

Figure 4. A: Hematoxylin and Eosin staining of the patient's resected left lacrimal gland mass showing ACC. B: Immunohistochemical staining of SV2A showing a negative result in the ACC, but a positive result in normal brain tissue.

istered in clinical trials. Imatinib was investigated in several studies, because the overexpression of c-kit is common in ACC. However, the use of this molecular-targeted drug resulted in no objective responses (8-10). Overall, few regimens with cytotoxic agents or molecular-targeted therapies have resulted in objective responses.

Levetiracetam binds to synaptic vesicle protein $2 \mathrm{~A}$ (SV2A) and is thought to decrease neuronal excitability, because the knockout of SV2A in mice leads to seizures (11). To investigate the mechanism underlying the anti-tumor effect of levetiracetam, in the present case we used a resected left lacrimal gland specimen from the patient and performed additional immunohistochemical staining of SV2A (mouse monoclonal anti-SV2A antibody 15E11; Abcam, Cambridge, UK). The immunohistochemical stains demonstrated that the patient's ACC was negative for SV2A (Fig. 4).

As for malignant tumors, the expression of SV2A has been observed frequently in gastrointestinal stromal tumors (GISTs) and glioma, but an anti-tumor effect of levetiracetam in malignant tumors expressing SV2A has never been reported (12). There has been no report of the expression of SV2A in ACC. With regard to the anti-tumor activity of levetiracetam, a case of glioblastoma multiforme (which did not include an examination of SV2A) that regressed with levetiracetam and dexamethasone was reported (13). Our present patient showed continuous regression of the tu- 
mor while on levetiracetam without any systemic cancertargeted therapy, corticosteroids or folk/home remedies, suggesting that the response was due to the levetiracetam. No prior case of ACC regression with levetiracetam treatment has been reported. Although the mechanism underlying the anti-tumor activity of levetiracetam remains unclear, our patient showed a favorable response to levetiracetam, and the anti-tumor effect was maintained over a long period.

To our knowledge, we have presented the first case of metastatic ACC showing a favorable response to levetiracetam. Further investigations are required to determine the precise indications and ultimate efficacy of levetiracetm in ACC.

The authors state that they have no Conflict of Interest (COI).

\section{References}

1. Dodd RL, Slevin NJ. Salivary gland adenoid cystic carcinoma: a review of chemotherapy and molecular therapies. Oral Oncol 42: 759-769, 2006.

2. Fordice J, Kershaw C, El-Naggar A, Goepfert H. Adenoid cystic carcinoma of the head and neck: Predictors of morbidity and mortality. Arch Otolaryngol Head Neck Surg 125: 149-152, 1999.

3. Stell PM. Adenoid cystic carcinoma. Clin Otolaryngol Allied Sci 11: 267-291, 1986.

4. Laurie SA, Ho AL, Fury MG, Sherman E, Pfister DG. Systemic therapy in the management of metastatic or locally recurrent adenoid cystic carcinoma of the salivary glands: a systematic review. Lancet Oncol 12: 815-824, 2011.

5. Van Herpen CM, Locati LD, Buter J, et al. Phase II study on gemcitabine in recurrent and/or metastatic adenoid cystic carci- noma of the head and neck (EORTC 24982). Eur J Cancer 44: 2542-2545, 2008.

6. Laurie SA, Siu LL, Winquist E, et al. A phase II study of platinum and gemcitabine in advanced salivary gland cancers: a trial of the NCIC Clinical Trials Group. Cancer 116: 362-368, 2010.

7. Hiraga S, Yiyoshi Y, Kishimoto S. Successful treatment of multiple pulmonary metastases of adenoid cystic carcinoma of the external auditory meatus with TS-1. Gan To Kagaku Ryoho 31: 1115-1117, 2004 (in Japanese, Abstract in English).

8. Ochel HJ, Gademann G, Rocken C, Wordehoff H. Effects of imatinib mesylate on adenoid cystic carcinomas. Anticancer Res 25: 3659-3664, 2005.

9. Hotte SJ, Winquist EW, Lamont E, et al. Imatinib mesylate in patients with adenoid cystic cancers of the salivary glands expressing c-kit: a Princess Margaret Hospital phase II consortium study. J Clin Oncol 23: 585-590, 2005.

10. Pfeffer MR, Talmi Y, Catane R, Symon Z, Yosepovitch A, Levitt M. A phase II study of Imatinib for advanced adenoid cystic carcinoma of head and neck salivary glands. Oral Oncol 43: 33-36, 2007.

11. de Groot M, Toering S, Boer K, et al. Expression of synaptic vesicle protein $2 \mathrm{~A}$ in epilepsy-associated brain tumors and in the peritumoral cortex. Neuro Oncol 12: 265-273, 2010.

12. Bumming P, Nilsson $\mathrm{O}$, Ahlman $\mathrm{H}$, et al. Gastrointestinal stromal tumors regularly express synaptic vesicle proteins: evidence of a neuroendocrine phenotype. Endocr Relat Cancer 14: 853-863, 2007.

13. Peddi P, Ajit NE, Burton GV, El-Osta H. Regression of a glioblastoma multiforme: spontaneous versus a potential antineoplastic effect of dexamethasone and levetiracetam. BMJ Case Rep 2016: 853-863, 2016

The Internal Medicine is an Open Access article distributed under the Creative Commons Attribution-NonCommercial-NoDerivatives 4.0 International License. To view the details of this license, please visit (https://creativecommons.org/licenses/ by-nc-nd/4.0/).

(C) 2018 The Japanese Society of Internal Medicine Intern Med 57: 721-724, 2018 